\title{
Changes to a Shift Reporting Sheet on a Critical Care Unit-Nurse Perceptions and Lessons Learned
}

This article was published in the following Dove Press journal: Journal of Multidisciplinary Healthcare

\author{
Rebecca McClay (D) \\ Jessica Natividad (iD ${ }^{2}$ \\ Michael Mileski iD ${ }^{3}$ \\ 'School of Science, Technology, \\ Engineering, and Math, American Public \\ University System, Charles Town, WV, \\ USA; ${ }^{2}$ Mother-Baby Unit, Midland \\ Memorial Hospital, Midland, TX, USA; \\ ${ }^{3}$ School of Health Administration, Texas \\ State University, San Marcos, TX, USA
}

Correspondence: Michael Mileski Texas State University, School of Health Administration, 60I University Drive, Encino Hall-250, San Marcos, TX, 78666, USA

Tel + I 5122453556

Email mileski@txstate.edu
Purpose: Improved perception and compliance with timely completion of the shift summary tool by bedside critical care nurses, and standardization of reported patient condition and treatment variables can be accomplished through collaboration and evidence-based modifications.

Materials and Methods: The IOWA Model was used as a framework to implement change in the population of practicing bedside nurses. In accordance with the AACN Healthy Work Environment standards, the population was given opportunity to complete surveys on a Likert scale to construct a best-fit instrument specific to the nurses' home units.

Results: Employee satisfaction with the report process increased, compliance with completion of the form increased, and satisfaction with consistency and facilitation was noted by nursing managers.

Conclusion: Focusing on frequently used items, or identifiable bottlenecks in communications, are likely to have the fastest and most impactful results for change. Workflows can be streamlined by using stakeholder input and feedback to clearly define the desired parameters and outcomes from the process.

Keywords: nursing, nursing perceptions, change of shift, critical care

\section{Introduction}

\section{Problem Description}

In the Critical Care Unit (CCU) of an acute care facility in West Texas, a paper shift summary tool is used to communicate patient acuity and specific needs through a bedside nurse written summary. The nurse completed tool is then used by the nursing managers as an aide for administrative duties. The paper format is used to facilitate portability and immediate changes such as admissions and patient changes. Administrative duties include communication between day and night shift, assigning oncoming shift staff, making admission bed assignments, and notification of patient safety concerns, such as restraint use and central line checks. Correct and timely information is important to these functions.

The original tool consisted of mostly open-ended questions which had limited standardized boxes about patient care, and a large open box for the nurse to fill in with more information. Subjectively, the summary tool caused negative feelings among the bedside nursing staff due to frustration and time consumption away from patient care. Objectively, the tool often did not get filled out in a timely manner and was frequently missing information desired by the nursing managers. 


\section{Available Knowledge}

A review of the literature demonstrated scholarly information addressing shift summary communication to management in critical care units was extremely limited. Literature on other acute care department communication methods were used to guide the evidence-based interventions of this project. ${ }^{1-3}$ Overall, little literature was available regarding nursing staff input into revision of systems and forms, or specific to the CCU.

\section{Rationale}

Allocation of staff and patient assignment is a complex and important tactical decision that should be supported with patient and nurse data through skilled communication and collaboration. ${ }^{3-5}$ Staffing models often reflect employee distribution and time requirements, but do not inform nurse managers about workload distribution, specialty conditions, and patient acuity management within a unit in real time. ${ }^{3}$ Literature is lacking regarding best measurements of the work of nursing and whether it should be measured in nurse to patient ratios, nursing staff required per patient day, total nursing hours per patient day, nurse perceived adequacy, and nursing skill to patient need matching. ${ }^{2}$ The tendency is for nursing managers to fall back on ratio and unit layout and patient location information to make patient assignments. ${ }^{6}$ Objective evaluation of patient acuity and needs has shown to improve nurse perception of assignments as more equal and more able to provide safe quality care. ${ }^{7}$ Requirements at the point of care are impacted by patient acuity and complexity of care are impacted by comorbidities, devices, and interventions such as titrations and wound care. ${ }^{2,4}$ Using a tool that skillfully and effectively communicates patient needs and bedside nursing care to nursing managers and bed board is essential for acuitybased assignments. ${ }^{1}$ Assignment balance depends on effective decision-making based on treatment requirements, nurse education on treatments, patient need for support in ADLs, medication frequency, and safe monitoring parameters. ${ }^{4,6}$ Finding an easy to use bridging tool to communicate patient conditions from the bedside to nursing managers is an important step toward achieving acuity-based assignments.

Evidence-based solutions for other units were found, specifically, a post-anesthesia care unit (PACU) used a hand off tool that indicated multiple patient conditions, complexity, and acuity information. ${ }^{1}$ However, the literature search yielded no CCU specific tools for this type of communication between bedside nurses and nursing manager positions.

\section{Specific Aims}

A paper communication tool had been in use within the local CCU to facilitate patient care and acuity information, essential for workflow and patient safety. The tool was found to consistently be inaccurate, incomplete, and poorly perceived by bedside staff who completed the tool based on empirical data collected and ultimately supported by initial survey results. Using a bedside nurse survey, the communication tool was identified as needing to be streamlined, inclusive of important measures, and improved for consistency across nurses. Collaborative efforts between bedside nurses and nursing managers led to creating a tool that accurately reflects acuity and nursing hours for a unit's CCU patients in a comprehensive and easy to use format. Changing to a standardized format using a charting by exception style was identified as a method to improve accuracy, bedside nurse perception, and timely completion of forms. ${ }^{1,8,9}$

\section{Purpose and Study Question}

The primary purpose of the intervention was to improve perception and timely completion of the paper shift summary tool by bedside critical care nurses, while increasing the completeness of reported patient conditions and treatment variables. Improving tool usability for nursing manager reporting and tracking of patient risk and safety was identified as a secondary purpose.

\section{Study Question}

Can changes be made to the current $\mathrm{CCU}$ nurse shift summary tool, that improve bedside nurse perception, and the usefulness of the information on the form, within six weeks after implementation?

\section{Model for Change}

The IOWA Model was used as a framework to implement change in the population of bedside nurses practicing in the author's institution CCU department. The trigger for change was the identification of both bedside nurse negative perceptions regarding the form and incomplete patient information being communicated. The organization deemed this a significant priority, pertaining to the mission of leading healthcare and the core value of pioneer spirit which supports exceeding expectations through teamwork. The team was formed by the principal investigator and included a nursing manager, an additional CCU bedside 
nurse, and a hospital representative from the evidencebased practice committee. The current literature was reviewed by the team, and a plan was made to adapt published evidence-based tools from other units to the hospital CCU. Initial rollout included bedside nurse and nursing manager education on the new tool and target outcomes for the innovation. Staff feedback at two weeks was incorporated into a revised version of the tool and follow-up was completed by a summative survey at six weeks. The tool was validated using follow-up with nursing managers and bedside nurses for feedback, and the tool was officially accepted by the CCU department head and entered the hospital's policy maintenance software.

\section{Materials and Methods}

\section{Context and Interventions}

The population of $\mathrm{CCU}$ nurses was given opportunity during two weeks for both day and night shifts to voluntarily complete an anonymous and confidential paper survey. Nineteen survey items were scored on a 5-point Likert scale ranging from "strongly disagree" to "strongly agree," and a blank area which was requested free text suggestions that participants had for form modifications. The Likert indicators were tallied and recorded, and a list was made of any written suggestions. Based on feedback from the surveys and published evidence, it was determined that changing to a charting by exception format that allowed bedside nurses to circle relevant conditions, treatments, and interventions was preferable to the open box form. ${ }^{1,11}$ Two nurses led the team that eventually added a nursing manager for a balanced approach. The new tool requiring nurses to circle identified acuity and nursing hour components halfway through their current shift was created in a spreadsheet software and trialed for two weeks.

\section{Study of the Interventions}

The initial staff survey sought information on nurse perception of the original tool including ease of use, time required to complete the tool, and potential patient characteristics that could be included for communication. The sample size for this survey was $n=23$. A survey at two weeks after the initial implementation of the new tool based on the original feedback and using the charting by exception format, was available to all CCU bedside nurses for 7 consecutive day and night shifts. Suggestions from these surveys led to additional changes to reporting components and spacing. The modified shift report tool was then trialed. Seven weeks after original, four weeks after the staff lead modifications, forms were implemented, a final survey closely mirroring the original survey was made available to the original population of CCU bedside nurses for a full two weeks. This final survey had a response sample size of $n=9$. The small sample size utilized for this manuscript is an acknowledged limitation but is likely related to the downturn in the oil industry which caused an exodus of nurses from the facility at the time of the investigation and the investigators' desire to only survey nurses that had used the original form. Actual sample sizes used in analysis demonstrate that staff nurses can take an existing tool, identify need for change, and implement a means for that change through authentic leadership and true collaboration at multiple levels within an organization. IRB approval was sought for the project and was awarded an exemption.

\section{Measures}

For the purposes of statistical discussion within this manuscript, responses were grouped to adjust for a lower $\mathrm{n}$ value in the final surveys. Included in one group is "strongly agree" and "agree" responses. To maintain conservative estimates "undecided," "disagree," and "strongly disagree" were all included in the second group. The use of these two groupings allowed for easier and more meaningful comparison of percentages to make better inferences from the results. Removing the "undecided" or having it as a zero measure was considered, however, due to the small sample size it was determined that it would be best to leave the numbers as assigned during the survey. SQUIRE 2.0 guidelines were utilized in the constructing of the manuscript and collection of the data. ${ }^{10}$

\section{Analysis}

The results are shown in Table 1 as pre and post percentages due to a significant change in the number of available staff to take part in the post survey due to oilfield volatility. Percentages were utilized to allow for an easier comparison between the pre and post numbers.

Table 2 shows information which was added to the revised instrument based on written responses from the first survey concerning what was missing from the initial instrument which was being used. These five areas were added to the revised instrument and perceptions were measured regarding their perceived importance, with none showing any less than a $75 \%$ importance rate, and three of these showing importance perceived by $100 \%$ of nursing staff overall. 
Table I Pre and Post Data Percentages from the Nursing Survey

\begin{tabular}{|c|c|c|}
\hline \multicolumn{3}{|l|}{ Baseline and Summative Data Positive Percentages } \\
\hline Survey Question & Pre & Post \\
\hline I. I have received training to fill out the shift report sheet properly. & $60.9 \%$ & $88.9 \%$ \\
\hline 2. Assignments are fairly made, according to patient acuity levels. & $21.7 \%$ & $77.8 \%$ \\
\hline 3. My assignments reflect my training level. & $59.1 \%$ & $87.5 \%$ \\
\hline 4. The shift report sheet is a waste of time. & $39.1 \%$ & $22.2 \%$ \\
\hline 5. The shift report sheet is clear and concise. & $21.7 \%$ & $66.7 \%$ \\
\hline 6. Filling out the shift report sheet takes valuable time away from taking care of my patient(s). & $47.8 \%$ & $11.1 \%$ \\
\hline 7. Listing the nurse and the Cisco number is vital information. & $65.2 \%$ & $66.7 \%$ \\
\hline 8. Listing the patient's diagnosis helps to make patient assignments. & $78.3 \%$ & $88.9 \%$ \\
\hline 9. Listing the age of the patient is vital information. & $45.8 \%$ & $88.9 \%$ \\
\hline 10. Listing the patient's doctor is vital information. & $69.6 \%$ & $88.9 \%$ \\
\hline II. Listing the patient's isolation status is vital information. & $87.0 \%$ & $88.9 \%$ \\
\hline 12. Listing if the patient has had a bath is vital information. & $20.8 \%$ & $75.0 \%$ \\
\hline 13. Listing the patient's code status is vital information. & $88.9 \%$ & $100.0 \%$ \\
\hline 14. Listing if the patient has restraints is vital information. & $91.3 \%$ & $100.0 \%$ \\
\hline 15. Overall, I am confident in filling out the shift report sheet. & $76.0 \%$ & $100.0 \%$ \\
\hline 16. The shift report sheet needs to be updated/The shift report sheet better fits my needs. & $65.2 \%$ & $88.9 \%$ \\
\hline 17. I enjoy filling out the shift report sheet. & $8.7 \%$ & $55.6 \%$ \\
\hline
\end{tabular}

Table 2 Information Added to New Instrument Based on Survey Results

\begin{tabular}{|l|c|}
\hline \multicolumn{2}{|l|}{ Summative Data Positive Percentages } \\
\hline Survey Question & Post (n=9) \\
\hline I. Listing types of patient drains is vital information. & $100.0 \%$ \\
2. Listing types of patient diet is vital information. & $75.0 \%$ \\
3. Listing types of patient voiding is vital information. & $100.0 \%$ \\
4. Listing the patient neuro status is vital information. & $100.0 \%$ \\
5. Indicating the patient acuity is vital information. & $88.9 \%$ \\
\hline
\end{tabular}

Appendix 1 includes the final CCU shift reporting sheet which resulted from this study.

\section{Ethical Considerations}

This test of change was implemented as a quality improvement project and was approved by the hospital Institutional Review Board as not qualifying to require their oversight. With this exemption, the initial survey began. Employed CCU nurses could choose to take part in any or all of the three anonymous surveys: initial, 2-week input, and final. No tracking or identifying information was collected on any of these surveys.

\section{Results}

Based upon survey results, satisfaction with the report process increased. Increased compliance with completion of the tool, and comments on additional unintended benefits of the format regarding consistency and report facilitation was noted on surveys completed by nursing managers.

The improvements which have been seen with the use of the improved form are apparent not only by nurses but also by nursing managers. The following statement included is from a nursing manager and her perceptions on the use of the new instrument:

The end of shift report sheet has been a tool our critical care unit has always utilized. This document has always been a task that nursing managers and nurses avoided using and never wanted to fill out. This form is very important for the leadership team when creating shift assignments. Since the creation of the new form and the ease of filling this form out, the leadership team has seen correct information being filled out, more nurses complying with the process, and a timelier turn around when getting the form back. As a nurse manager I utilize this form for more than shift assignments, I can utilize this form for month end audits for our critical care unit, because of the new format the audits are less time consuming and not so difficult to complete.

There was a significant difference between perceptions of the previously used tool and the tool which was implemented with staff input. Some of the more significant areas of Table 1 are quoted from bedside nurses below.

\#1-I have received training to fill out the shift report sheet properly. Between pre and post, there was a $28 \%$ 
difference in the staff perceptions in this area. It becomes obvious that staff training on any instrument is not only key to its successful use but also to staff buy-in to the use of the new instrument as well.

\#2-Assignments are fairly made, according to patient acuity levels. Between pre and post, there was a $56.1 \%$ difference in the perceptions of the staff in this area. The floor nurse perception was that acuity was not being used to make assignments for the staff before the use of the new instrument. Intriguingly, still only $77.8 \%$ view the instruments use to fairly assign patients based on acuity levels. This percentage allows for an opportunity for further investigation overall.

\#3-My assignments reflect my training level. Between pre and post, there was a $28.4 \%$ difference. $28.4 \%$ of staff had an increased perception that their assignment was now more aligned to their training level than it was with the previous instrument being used. While alignment between administration and staff in this area improved, there is still room for growth.

\#4-The shift report sheet is a waste of my time. Between pre and post, demonstrated an interesting situation. For pre, only $39.1 \%$ stated that the shift report sheet was a waste of their time, which overall shows that most nurses understand the need for the sheet and how important it is for it to be provided to administration. For post, the number decreased to $22.2 \%$, showing that the changes made to the sheet were positively perceived by the staff overall.

\#5-The shift report sheet is clear and concise. Between pre and post, there is a $45 \%$ difference of staff perceptions. Only $21.7 \%$ thought the sheet was clear and concise before changes. Perceptions improved to $66.7 \%$ of respondents viewing the sheet as clear and concise, demonstrating the importance of staff input in for effective instrument development.

\#6-Filling out the shift report sheet takes valuable time away from taking care of my patient(s). Between pre and post change, there is a $36.7 \%$ difference, with an improvement of $47.8 \%$ pre to only $11.1 \%$ post feeling that patient care was affected by the tool. The value of the instrument changes clearly demonstrated a positive shift in nursing perception.

\#9-Listing the age of the patient is vital information. It is unclear shy there is a decreased perception of the importance of patient age by $43.1 \%$ from pre to post survey. Perhaps, the new instrument allowed nursing staff to see and understand the patient in a much better light, allowing them to not only see clinical issues but also how the age of the patient fit into this, allowing for more appropriate assignments.

\#12-Listing if the patient has had a bath is vital information. From pre to post there was a $54.2 \%$ change in perception overall. Of note, $75 \%$ of nurses post assessment thought that bath status was of great importance. Potentially alluding to the efficacy of the instrument itself allowing staff to begin to switch their focus to more customer service-oriented care.

\#13/14 - Listing the patient's code status is vital information/Listing if the patient has restraints is vital information. Nurse perception increased to $100 \%$ of nurses surveyed believing that knowing that these areas are of vital importance. Showing a change in the perceptions of the staff to more of a patient focus based on the rollout of the new instrument.

\#15-Overall, I am confident in filling out the shift report sheet. Between pre and post here there was a $24 \%$ change to $100 \%$ of nurses being confident in filling out the shift report sheet after the implementation of the new format. Inferring that nursing staff now has a much better understanding of what is expected to fill out the sheet and how impactful their contributions can be.

\#17-I enjoy filing out the shift report sheet. From pre to post there was a $46.9 \%$ increase from $8.7 \%$ to $55.6 \%$ of staff with a perception of enjoying filling out this sheet. Indicating that the time required to include bedside nurse feedback is warranted and influences perception toward the tool allowing them to provide better overall care to their patients.

\#8/10/13 - Listing the patients diagnosis helps to make patient assignments/Listing the patient's doctor is vital information/Listing the patient's isolation status is vital information. In each of these areas there was an increased positive perception showing an increased staff understanding of why this information is important to the provision of care and in the assignment of patients to staff.

\section{Discussion}

\section{Summary}

The key finding for this study was significantly improved nurse perception of a CCU communication tool using the application of evidence-based charting principles and bedside nurse collaboration and evaluation throughout the change. A secondary key finding was the improved ability of nursing managers to use the portable tool for appropriate 
staffing assignments and tracking of nursing care changes. These findings are based on analysis and comparison of survey instrument questions and free text responses. The strength of the project lies in authentic leadership of bedside nurses facilitating multilevel communication while improving nurse perception of a necessary tool by implementing true collaboration of invested staff members.

\section{Interpretation}

\section{Limitations}

One limitation of this study is the limited numbers of staff available for the duration of the study. The number of bedside nurses on a single critical care unit is already limited, however, during data collection for this study, there was a shift in numbers of the target population due to oilfield employment volatility. As such the survey $n=$ decreased from 23 pre to 9 post. This significantly smaller post-n had the potential to cause issues with statistical validity, however, the empirical results of this study were significant overall.

\section{Conclusion}

The importance of including bedside nurse participation in the implementation team is important, and one that has been outlined in other papers. ${ }^{12}$ Significant changes in perceptions occurred regarding the use of the shift change report from the initial communication tool to the revised new tool. Likert scale percentages clearly showed differences in perception, usability, completeness, and accuracy of the communicated patient information. Including the floor nurses in the process throughout appeared to increase buy-in of the unit staff, encourage feedback at all follow-up opportunities, and created a greater understanding of the instrument, its uses, and its importance. While not all measured outcomes demonstrated significant change, those that did were impressive in magnitude.

\section{Future Directions}

During this implementation, it became apparent that the use of a tool in this manner could be applied to other units. Work within the hospital to expand use of the communication tool, adapting to patient population conditions and treatments, while standardizing as much as possible. The tool has already been trialed in another unit already with successful results. The tool is currently being considered as a step in making a template for electronic communication within the current electronic health record software.

\section{Ethics Disclosure}

This work was exempted from normal IRB processes by Midland Memorial Hospital, Midland, Texas. It was considered a "quality improvement" project by the facility. The research was conducted in accordance with the Declaration of Helsinki.

\section{Disclosure}

The authors report no conflicts of interest in this work.

\section{References}

1. Halfpap E. Staff developed PACU acuity scoring grid. $J$ Perianesth Nurs. 2016;31(4):303-308. doi:10.1016/j.jopan.2014.09.008

2. Min A, Scott LD. Evaluating nursing hours per patient day as a nurse staffing measure. J Nurs Manag. 2016;24(4):439-448. doi:10.1111/ jonm. 12347

3. Sir MY, Dundar B, Barker Steege LM, Pasupathy KS. Nurse-patient assignment models considering patient acuity metrics and nurses' perceived workload. $J$ Biomed Inform. 2015;55:237-248. doi:10.1016/j.jbi.2015.04.005

4. American Association of Critical Care Nurses. Healthy work environments. 2020. Retrieved from: https://www.aacn.org/nursingexcellence/healthy-work-environments. Accessed February 11, 2021.

5. Institute of Medicine. To Err is Human: Building a Safer Health System. Washington, DC: The National Academies Press; 2000. doi: $10.17226 / 9728$

6. Thomas E, Brathwaite EE, Cohn T, Nerey J, Lindgren CL, Williams S. Clinical partners' perceptions of patient assignment according to acuity. Med Surg Nurs. 2015;24(1):39-45.

7. Morales D, Winchester C. Objective postpartum acuity tool provides equitable nurse-patient assignments. J Obstet Gynecol Neonatal Nurs. 2019;48(3):S8. doi:10.1016/j.jogn.2019.04.013

8. Lim F, Pajarillo E. Standardized handoff report form in clinical nursing education: an educational tool for patient safety and quality of care. Nurse Educ Today. 2016;37:3-7. doi:10.1016/j. nedt.2015.10.026

9. Ronald D, Fielding P, Davies F. Novel use of a children's emergency department scoring system as a marker of resource utilisation. Emerg Med J. 2015;32(12):1009.2-1010. doi:10.1136/emermed-2015205372.69

10. Ogrinc G, Davies L, Goodman D, Batalden PB, Davidoff F, Stevens D. SQUIRE 2.0 (Standards for Quality Improvement Reporting Excellence): revised publication guidelines from a detailed consensus process. BMJ Qual Saf. 2015;2015.

11. Ulrich B, Barden C, Cassidy L, Varn-Davis N. Critical care nurse work environments 2018: findings and implications. Crit Care Nurse. 2019;39(2):67-84. doi:10.4037/ccn2019605

12. McClay R, Mileski M. Don't roll your eyes, roll out change. Nurs Made Incred Easy. 2019;17(6):52-54. doi:10.1097/01.NME. 0000585096.42678 .39 


\section{Publish your work in this journal}

The Journal of Multidisciplinary Healthcare is an international, peerreviewed open-access journal that aims to represent and publish research in healthcare areas delivered by practitioners of different disciplines. This includes studies and reviews conducted by multidisciplinary teams as well as research which evaluates the results or conduct of such teams or healthcare processes in general. The journal covers a very wide range of areas and welcomes submissions from practitioners at all levels, from all over the world. The manuscript management system is completely online and includes a very quick and fair peer-review system. Visit http://www.dovepress.com/testimonials. php to read real quotes from published authors.

Submit your manuscript here: https://www.dovepress.com/journal-of-inflammation-research-journal 